\title{
New insights into onset mechanisms of atrial fibrillation and flutter after coronary artery bypass graft surgery
}

\author{
A D Taylor, J G Groen, S L Thorn, C T Lewis, A J Marshall
}

Heart 2002;88:499-504

See end of article for authors' affiliations

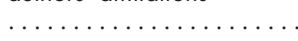

Correspondence to: Dr A D Taylor, Cardiology Department, The South West Cardiothoracic Centre, Derriford Hospital, Plymouth PL6 8DH, UK; dradtaylor@supanet.com

Accepted 4 July 2002

\begin{abstract}
Objective: To determine by means of a monitoring study the onset mechanisms of atrial fibrillation (AF) after coronary artery bypass graft surgery (CABG).

Patients and design: During elective CABG, 81 patients had one bipolar atrial and one ventricular epicardial electrode attached. These were connected to a Vitatron $900 \mathrm{E}$ external pacing device, which monitored the patients for four consecutive days. 12 lead ECGs were obtained if AF was clinically detected and Holter ECGs were obtained in $8(33 \%)$ of these patients.

Results: 24 patients $(30 \%)$ developed paroxysmal AF (50\%), atrial flutter $(17 \%)$, or both $(33 \%)$. The number of AF episodes varied from 1-169 a day (median 5) and were usually of short duration (median 2.25 minutes). Pacemaker diagnostics showed much intrapatient and interpatient variability in onset mechanisms but the majority of AF onsets $(71 \%)$ were preceded by either short runs of AF or multiple atrial extrasystoles. The final trigger was a conducted atrial extrasystole in $72 \%$ of cases. There were no bradycardic triggers. The Holter ECGs confirmed the device's data.

Conclusions: The onset mechanisms of post-CABG AF are dominated by atrial extrasystoles with multiple atrial extrasystoles and short runs of AF preceding the main AF onset in the majority of cases. These results have major implications for the development of new preventive pacing algorithms.
\end{abstract}

$\mathrm{P}$ ostoperative atrial fibrillation (AF) and flutter (AFL) remain the most common arrhythmic complications of coronary artery bypass graft surgery (CABG), occurring in about $30 \%$ of cases with a peak incidence on days $2-3$ postoperatively. ${ }^{1-6}$

Although AF after CABG is usually self limiting and not associated with significant morbidity, it can cause haemodynamic compromise and increase the risk of postoperative stroke. ${ }^{78}$ Hospital stay may be prolonged resulting in significant increases in costs. ${ }^{23}$ Prophylactic drug treatment with $\beta$ adrenergic antagonists, sotalol, and amiodarone have been shown to reduce the incidence of postoperative AF. ${ }^{9-13}$ These treatments may be limited by side effects and are contraindicated in some patients. In addition there remains a significant incidence of AF despite such treatment: $22 \%$ with oral amiodarone and $\beta$ blocker combined. ${ }^{13}$

Alternative non-pharmacological strategies for AF prevention are required. Recently the results of several trials of epicardial single and dual site atrial pacing (with a variety of pacing algorithms) have been published with generally favourable results. ${ }^{14-20}$ However, a prerequisite to the development of an optimal pacing algorithm is a detailed understanding of the AF onset mechanisms in this post-surgical population. The aim of the present monitoring study was to determine the frequency, duration, temporal patterns, and onset mechanisms of AF after CABG to guide the design of an optimal atrial based pacing treatment for the prevention and termination of AF.

\section{METHODS}

\section{Patient population}

One hundred consecutive patients scheduled for elective CABG were screened for participation in the study. Inclusion criteria were first elective CABG (requiring cardiopulmonary bypass) for stable angina with no age limit and sinus rhythm preoperatively. Exclusion criteria were additional cardiac surgery, previous documented AF, concurrent antiarrhythmic treatment other than $\beta$ blockers (but excluding sotalol) and calcium channel blockers used for angina and hypertension, permanent pacemaker implantation, and a left ventricular ejection fraction measured echocardiographically of $<40 \%$. All patients had a preoperative ECG and transthoracic echocardiogram. Patients were selected with normal or mildly depressed left ventricular function only, since these form the majority of candidates for elective CABG and severe left ventricular dysfunction in itself may predispose to AF. The local ethics committee approved the study and all patients signed an informed consent before participating.

\section{Study protocol}

All patients underwent isolated CABG on standard cardiopulmonary bypass with myocardial protection provided by cardioplegia. During surgery all patients had one atrial and one ventricular bipolar epicardial electrode (Medtronic 6495, Medtronic Inc, Minneapolis, Minnesota, USA) attached to the muscular part of the right atrium, close to the interatrial groove and right ventricular myocardium, respectively.

On return to the cardiothoracic intensive care unit an external Vitatron Selection 900E pacemaker (Vitatron Medical, Dieren, Netherlands) was attached to the electrodes. The device was programmed with atrial and ventricular sensitivities of $0.25 \mathrm{mV}$ and $1.0 \mathrm{mV}$, respectively, and with the atrial arrhythmia diagnostic function enabled. The atrial blanking period was set at $50 \mathrm{~ms}$ and the atrial arrhythmia detection rate at 200 beats/min. Atrial and ventricular outputs, pulse widths, and lower rate limit were programmed to their minimum values to preclude pacing. If rescue pacing was required, the device was removed and a temporary pacing unit was attached to the epicardial leads.

After fine tuning of the diagnostic parameters in the first 10 patients, the number of ventricular beats to detect the onset of

Abbreviations: AF, atrial fibrillation; AFL, atrial flutter; AFT, atrial fibrillation therapy; $C A B G$, coronary artery bypass graft surgery; $D O R$, detailed onset report; PAF, paroxysmal atrial fibrillation 
Table 1 Baseline patient characteristics

\begin{tabular}{|c|c|c|c|c|}
\hline Variable & All patients $(n=81)$ & Non-AF $(n=57)$ & $\mathrm{AF}(n=24)$ & $\mathrm{p}$ Value \\
\hline \multicolumn{5}{|l|}{ Preoperative data } \\
\hline Age (years) & $62(8)$ & $60.7(8.8)$ & $67(6)$ & 0.007 \\
\hline Heart rate (beats/min) & $61(12)$ & $62(12)$ & 59 (13) & 0.15 \\
\hline Male sex & 72 (89\%) & $50(88 \%)$ & $22(92 \%)$ & 0.72 \\
\hline $\operatorname{LVEF}(\%)$ & 55 (16) & 55 (15) & $56(18)$ & 0.76 \\
\hline Left atrial size $(\mathrm{cm})$ & $4.0(0.7)$ & $3.9(0.7)$ & $4.1(0.7)$ & 0.17 \\
\hline \multicolumn{5}{|l|}{ Preoperative medication } \\
\hline$\beta$ Blocker stopped on day of surgery & $19(23 \%)$ & $15(26 \%)$ & $4(17 \%)$ & 0.28 \\
\hline$\beta$ Blocker continued & $31(38 \%)$ & $20(35 \%)$ & $11(46 \%)$ & 0.28 \\
\hline Calcium antagonist & $38(47 \%)$ & $29(51 \%)$ & $9(38 \%)$ & 0.27 \\
\hline Nitrates & $36(44 \%)$ & $21(37 \%)$ & $15(63 \%)$ & 0.03 \\
\hline Statin & $67(83 \%)$ & $45(79 \%)$ & $22(92 \%)$ & 0.21 \\
\hline ACEI & $14(17 \%)$ & $12(21 \%)$ & $2(8 \%)$ & 0.21 \\
\hline \multicolumn{5}{|l|}{ Medical history } \\
\hline COPD & $2(2 \%)$ & $1(2 \%)$ & $1(4 \%)$ & 0.51 \\
\hline Diabetes & $10(12 \%)$ & $6(11 \%)$ & $4(17 \%)$ & 0.47 \\
\hline Hypertension & $34(42 \%)$ & $25(44 \%)$ & $9(38 \%)$ & 0.60 \\
\hline Myocardial infarction & $28(35 \%)$ & $20(35 \%)$ & $8(33 \%)$ & 0.88 \\
\hline \multicolumn{5}{|l|}{ Perioperative data } \\
\hline Number of vessels grafted & $3.1(0.8)$ & $3.1(0.8)$ & $3.0(0.9)$ & 0.97 \\
\hline LIMA & $77(85 \%)$ & $56(89 \%)$ & $21(75 \%)$ & 0.08 \\
\hline RIMA & $1(1 \%)$ & $1(2 \%)$ & 0 & 1.00 \\
\hline Radial & $2(2 \%)$ & $2(4 \%)$ & 0 & 1.00 \\
\hline Aortic cross clamp time (min) & $39(43)$ & $38(40)$ & $43(50)$ & 0.61 \\
\hline Bypass pump time (min) & 68 (29) & $65(26)$ & $74(35)$ & 0.29 \\
\hline
\end{tabular}

Data are mean (SD) unless otherwise indicated.

$\mathrm{ACEI}$, angiotensin converting enzyme inhibitor; AF, atrial fibrillation; COPD, chronic obstructive pulmonary disease; LIMA, left internal mammary artery; LVEF, left ventricular ejection fraction; RIMA, right internal mammary artery.

an atrial arrhythmia episode was set to 15 and beats to detect offset was set to 30 . The pacemaker mode was programmed to DDD. A maximum of six detailed onset reports (DORs) of AF onsets can be stored each day (one DOR is a beat to beat recording of atrial and ventricular events around the onset of $\mathrm{AF}$ ) with these device settings. We chose to program the device to detect all episodes of AF of 15 ventricular beats or more so that we could obtain as much detail as possible about the onset mechanisms of these longer (and probably more clinical relevant) AF episodes. These onset mechanism data are recorded only in the DOR. However, the arrhythmia counters recorded all episodes of AF (of 15 ventricular beats or more) and the arrhythmia diaries gave the times of occurrence and exact durations of up to 32 episodes of AF a day.

The patients were monitored continuously with the device for four days postoperatively. Data were downloaded each day on to a diskette and analysed with an AF Discover 1 (Vitatron Medical) program. A 12 lead ECG was obtained if the patient developed AF/AFL and daily thereafter. Patients' symptoms, daily urea and electrolytes, and discharge day were recorded. Intravenous or oral potassium supplements were given to maintain serum potassium concentrations of 4-5 mmol/l for the duration of the study. Pharmacotherapy for AF/AFL and the decision whether to continue with preoperative $\beta$ blockade was left to the attending physician or surgeon. Of the 24 patients who developed AF/AFL, 8 (33\%) had a 24 hour ECG to compare with the device's recorded data.

AF was diagnosed on the total irregularity of the interatrial intervals (shown on the ECG marker diagrams), which were generally $<200 \mathrm{~ms}$, and on the irregular ventricular response. AFL was diagnosed on regular interatrial intervals of about $200 \mathrm{~ms}$ and, in this study, the invariable 2:1 atrioventricular conduction resulting in ventricular response rates of about 150 beats/min. In addition the 12 lead and 24 hour ECGs confirmed the rhythm.

\section{Outcome measures}

The primary end point was the occurrence of episodes of AF/AFL (of any length) detected by the device so that onset mechanisms, duration, temporal patterns, and frequency could be determined.

\section{Statistical analysis}

Statistical analyses were by two tailed Mann-Whitney test for continuous data and $\chi^{2}$ test for categorical data. Statistics were calculated using software (SPSS version 9.0, SPSS Inc, Chicago, Illinois, USA). Significance was considered at $\mathrm{p}<0.05$. Values are given as mean $(\mathrm{SD})$ or proportions (percentages) and median values are stated.

\section{RESULTS}

\section{Patient characteristics}

A total of 81 patients (62 (8) years old, 72 (89\%) male patients) met the inclusion criteria and consented to participate in the study. Table 1 shows baseline patient characteristics. No patient had significant left atrial enlargement or valve disease. The proportion of pre- and postoperative use of $\beta$ blockers was non-significant between the non-AF and AF groups. The only significant differences between the groups in baseline characteristics were age and the use of oral nitrates. All patients who developed AF had a serum potassium concentration between $4-5 \mathrm{mmol} / \mathrm{l}$.

\section{Complications}

Postoperative complications were small and there were no deaths. There were no complications related to epicardial lead insertion or removal.

\section{Occurrence, frequency, and duration of AF/AFL episodes}

Twenty four patients (30\%, 92\% male patients) developed paroxysmal AF (PAF) $(50 \%)$, AFL ( $17 \%)$, or both (33\%). PAF was detected clinically and confirmed by 12 lead ECG in 18 patients. The four patients who developed AFL were all in the clinically detected group and had 2:1 atrioventricular conduction with ventricular rates around 150 beats/min and atrial rates of 300 beats/min. This was confirmed by 12 lead ECG, the rate profile diagrams, and the interatrial intervals of about $200 \mathrm{~ms}$ on the ECG marker diagrams contained within the DOR.

The number of AF/AFL episodes (in the arrhythmia counters section of the device's recorded data) varied from 1-169 a day with a median of 5 (mean $10(11))$. The durations 
Table 2 Number of AF episodes and restarts within 1 and 5 minutes

\begin{tabular}{llll}
\hline & $\begin{array}{l}\text { Total } \\
\text { episodes } \\
\text { of AF }\end{array}$ & $\begin{array}{l}\text { Restarts } \\
\text { within 1-5 } \\
\text { minutes }\end{array}$ & $\begin{array}{l}\text { Restarts } \\
\text { within 1 } \\
\text { minute }\end{array}$ \\
\hline $\begin{array}{l}\text { Non-clinical AF }(n=6) \\
\text { Clinical AF }(n=18)\end{array}$ & 377 & $8(15 \%)$ & $18(33 \%)$ \\
Combined $(n=24)$ & 431 & $108(29 \%)$ & $182(48 \%)$ \\
\hline
\end{tabular}

of the AF/AFL episodes (from the arrhythmia diaries) were similarly variable from 6 seconds to 18.4 hours (mean 44 minutes (1.5) hours; median 2.25 minutes) for the 24 patients combined. However, the patients with AF detected only by a device had a lower duration range of 6 seconds to 22.3 minutes (median 1 minute) compared with 6 seconds to 18.4 hour (median 2.5 minutes) in the 18 patients with clinically detected AF.

There was no correlation between the frequency and duration of PAF or AFL episodes and the pharmacological agent used to treat the patient. In addition, in patients with frequent episodes of PAF, there was no increase in duration of the episodes as their number increased. Episodes lasting a few seconds and those lasting over an hour were scattered at random throughout each 24 hour recorded period.

\section{Temporal patterns and diurnal distribution of AF/AFL episodes}

The arrhythmia diaries were analysed for the number of AF restarts within one minute and five minutes. These are shown for the six patients with non-clinically detected AF, the 18 patients with clinically detected AF, and the two groups combined in table 2 . The AF episodes were significantly predominant from 0130-1030 ( $\mathrm{p}=0.023)$. Eighty six per cent of all AF/AFL episodes occurred on days 3 and 4 postoperatively, $13 \%$ on day 2 , and $1 \%$ on day 1 .

\section{Onset mechanisms of AF/AFL}

We defined onset mechanisms as the arrhythmic events in the 30 seconds leading up to the AF/AFL onset plus the final trigger (the last complex before AF/AFL onset). Atrial lead undersensing (24\%) and far field $\mathrm{R}$ wave detection (7\%) degraded $31 \%$ of the atrial lead recorded data. However, this was easy to recognise and rigorously excluded from the final analysis to leave only clean data.

Eighty nine DORs from 22 patients were useful, detailing the final trigger and arrhythmic events leading to AF/AFL onset. Within the 89 DORs there were $151 \mathrm{PAF} / \mathrm{FL}$ episodes. This included $62(41 \%)$ short runs of AF (4-25 atrial beats) that occurred before the main AF episode onset (fig 1).

From the DORs the final trigger (for all 151 episodes) was identified as a conducted atrial extrasystole (fig 2) in 109 $(72.2 \%)$, a ventricular extrasystole in $1(0.7 \%)$, and "no trigger" (either a non-conducted atrial extrasystole or first sensed spontaneous AF potential) in 41 (27.1\%). Trigger combinations were rare, occurring in 8 of 89 DORs (in five patients); all were a conducted atrial extrasystole plus no trigger apart from one case of a conducted atrial extrasystole and a ventricular extrasystole. No patient had a bradycardic trigger.

The events leading to the main AF episode in the DORs were highly variable within and between patients, with the short runs of $\mathrm{AF}$ and multiple conducted atrial extrasystoles (including couplets, triplets, bigeminy, and trigeminy) predominating. Twenty nine of 89 (33\%) DORs contained short runs (mean 2 (1), which occurred in 9 of the AF patients) and there was no increase in the duration of the short runs before the main AF episode onset. The number of atrial extrasystoles

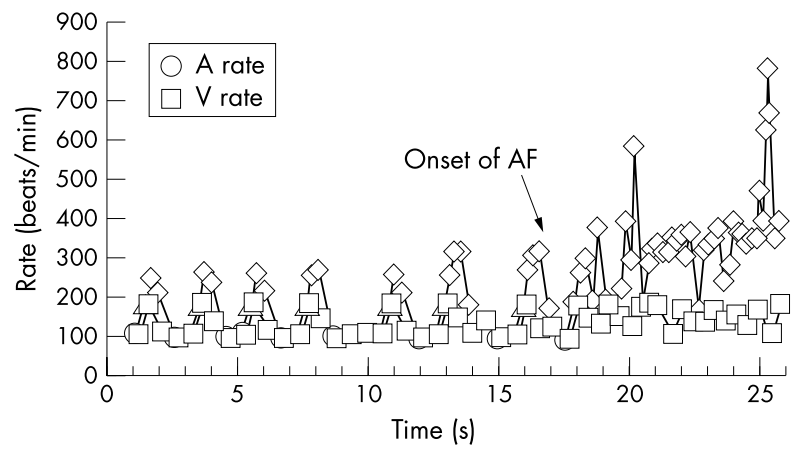

Figure 1 A detailed onset report containing atrial extrasystolic triplets and short runs of atrial fibrillation (AF) preceding the main AF onset. Diamonds indicate tachycardic atrial sensed events; triangles indicate isolated atrial extrasystole.

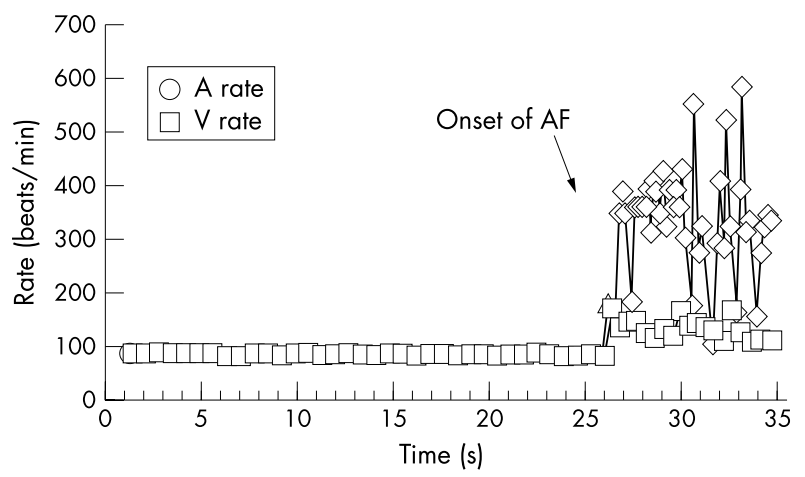

Figure 2 A detailed onset report showing a single conducted atria extrasystole precipitating AF. Diamonds indicate tachycardic atrial sensed events; triangles indicate isolated atrial extrasystole.

varied from 1-47 (mean 5 (7), median 3). Thirty four of 89 (38\%) DORs contained multiple atrial extrasystoles (in 10 patients) but no short runs. Twenty four of 89 (27\%) DORs contained just one conducted atrial extrasystole, which was the final trigger to the AF onset, and two DORs had just one no trigger main AF episode onset.

In total, 19 patients had short runs of AF or multiple atrial extrasystoles in some of their DORs but eight of these patients also had DORs containing just one (final trigger) atrial extrasystole. Three patients had no short runs or multiple atrial extrasystoles at all.

\section{Comparison of the number of atrial extrasystoles between the non-AF and AF groups}

The number of atrial extrasystoles a day in the non-AF and AF groups were compared. These data are recorded in the arrhythmia counters but, because the device labels only the first complex of an atrial extrasystole couplet/triplet as an atrial extrasystole, absolute numbers are not obtained but a trend can be seen. Table 3 shows the daily atrial extrasystole numbers for the two groups. A significantly greater number of atrial extrasystoles a day was seen in the AF group on days 3 and 4 , which is when $86 \%$ of all AF/AFL episodes occurred.

\section{Twenty four hour ECG data and ventricular response rate in AF}

Eight of 24 (33\%) patients who had AF episodes had a total of nine 24 hour ECGs on the days when they had AF. The tapes confirmed the frequency and duration of the AF episodes detected by the device. Some of the tapes also showed very short episodes of PAF of $<15$ (ventricular) beats not detected by the device since the beats to detect onset was programmed at 15 . 
Table 3 Number of atrial extrasystoles a day in the non-AF and AF groups

\begin{tabular}{|c|c|c|c|c|c|c|c|}
\hline \multirow[b]{2}{*}{ Day } & \multicolumn{3}{|l|}{$\mathrm{AF}$} & \multicolumn{3}{|l|}{ Non-AF } & \multirow[b]{2}{*}{$\mathrm{p}$ Value* } \\
\hline & Mean (SD) & Median & Range & Mean (SD) & Median & Range & \\
\hline 1 & $667(1716)$ & 80 & $18-7314$ & 305 (649) & 99 & $5-4022$ & 0.89 \\
\hline 2 & 620 (1551) & 81 & $9-6565$ & $544(1176)$ & 80 & $7-4765$ & 0.97 \\
\hline 3 & $1251(1334)$ & 646 & $5-4839$ & $520(1100)$ & 41 & $5-4637$ & 0.004 \\
\hline 4 & 1275 (1874) & 366 & $5-5563$ & 577 (953) & 54 & $7-3009$ & 0.014 \\
\hline
\end{tabular}

The rhythm (AF or AFL) was consistent with the device's data, as was the ventricular response rate. The final trigger was confirmed (fig 3) in $93.5 \%$ of cases; the discrepancy lay in some no trigger episodes on the DOR, which were probably conducted atrial extrasystole triggers on the 24 hour ECGs.

The mean ventricular response rates (obtained from the device's 24 hour Holter) were 117 (11) beats/min for the six patients with non-clinically detected AF, 137 (15) beats/min for the 18 patients with clinically detected $\mathrm{AF}$, and 134 (16) beats/min for the two groups combined. These rates were confirmed by the ECGs obtained from the patients with clinically detected AF and the 24 hour ECGs.

\section{Sinus rates in the preceding one minute to AF onset}

For the patients who developed $\mathrm{AF}$, the mean ventricular rate in the one minute to AF onset was estimated from the rate trend data shown on the DORs. We omitted those rates where the arrhythmia diaries showed an episode of AF in the last minute to the AF onset of that DOR (since this would have spuriously increased the ventricular rate). These data were corroborated by the 24 hour ECGs and the rate profile diagrams, which confirmed sinus rhythm before onset. There was variability but the mean sinus rate in the minute before AF onset was 99 (21) beats/min.

\section{Symptoms, treatment, and discharge day}

The most common symptoms in the 18 patients with clinically detected AF were palpitations and breathlessness. No patient developed haemodynamic compromise or required cardioversion.

Of the 18 patients with clinically detected AF, four were treated only with oral flecainide, one with oral flecainide and oral digoxin, five with digoxin only, two with digoxin and atenolol, one with digoxin and oral amiodarone, and three with intravenous amiodarone. Two patients had reverted to sinus rhythm before pharmacotherapy was commenced.

The discharge day in the non-clinical AF group was 6 (1.5) days, in the clinical AF group 8 (2.5) days, and in the non-AF group 6 (1.5) days. Length of stay was significantly prolonged for the clinical AF group compared with the non-AF patients $(\mathrm{p}=0.001)$.

\section{DISCUSSION}

As with previous studies ${ }^{1-5} 21$ we confirm age as the most reliable predictor of AF after CABG. The non-AF group had a mean age of 60.7 (8.8) years and the AF group 67 (6) years $(p=0.007)$ (table 1$)$. Aranki and colleagues ${ }^{3}$ suggested that age related changes in the atrial substrate (for example, dilatation, myocardial atrophy, and fibrosis) may be responsible. The significant $p$ value $(0.03)$ for the use of preoperative oral nitrates as a predictor for post-CABG AF is probably a chance finding (due to the small numbers of patients) and is not supported in the literature. The clinically detected AF group had significantly longer hospital stays than the non-AF group, consistent with previous published data. ${ }^{2}{ }^{14}$

Eighty six per cent of all AF/AFL episodes occurred 48-96 hours postoperatively, which appears to be the usual narrow time window for atrial arrhythmias after $\mathrm{CABG}^{4-622} \mathrm{~A}$ multifactorial pathogenesis for post-CABG AF has been suggested, with sterile pericarditis, high sympathetic drive, autonomic imbalance, atrial ischaemia, and delayed intraatrial conduction all implicated. ${ }^{623}$

Onset mechanisms of AF and AFL

Relatively little is known about the detailed onset mechanisms of $\mathrm{AF} / \mathrm{AFL}$ in the post-CABG population. It is generally

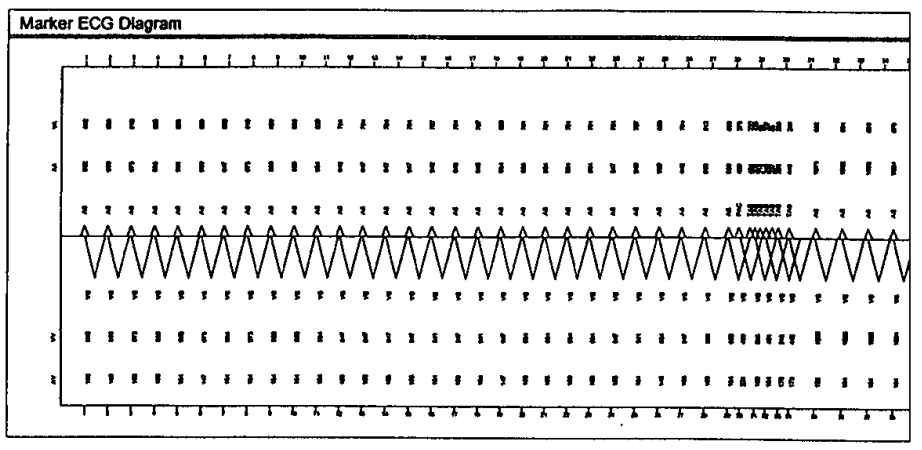

Figure 3 Part of a 24 hour ECG (bottom tracing) confirming the corresponding marker ECG diagram (top tracing), which shows a conducted atrial extrasystole precipitating $\mathrm{AF}$.

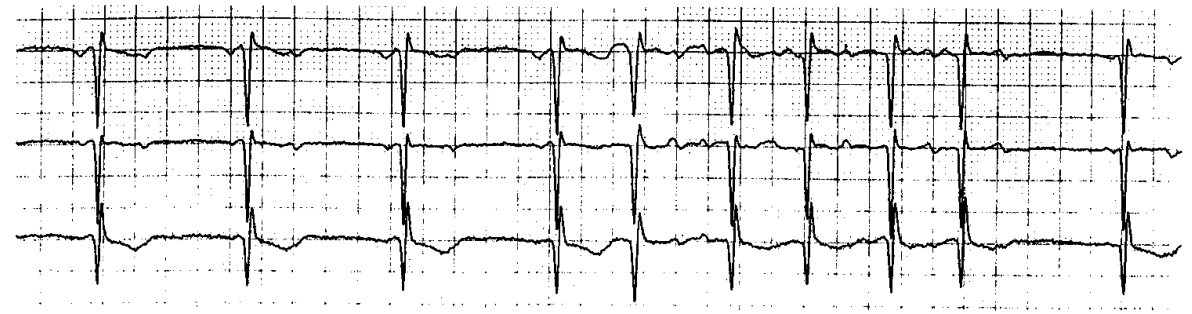


accepted that AF consists of multiple re-entrant circuits propagating throughout both the left and right atria, often initiated by an atrial extrasystole encountering areas of slow conduction and unidirectional block. ${ }^{24}{ }^{25}$ In the post-cardiac surgery population, prolonged atrial refractoriness leading to slow conduction and regions of block, together with predisposing age related electrophysiological changes in the atrial substrate, appears important. ${ }^{6}$

Blommaert and colleagues, ${ }^{17}$ in a trial of continuous atrial overdrive pacing for AF prevention after CABG, studied AF initiation by analysing Holter ECGs. Atrial extrasystoles were the precipitant of AF in the majority of cases and predominated in the five minutes preceding AF onset. Previous monitoring studies with either continuous ECG monitoring or 24 hour ECGs have not reported in detail the AF/AFL durations or onset mechanisms $s^{512627}$ and usually only clinically significant episodes of arrhythmia were reported. However, White and colleagues $^{26}$ reported a total of 291 episodes of AF or AFL in 41 patients, continuously monitored for seven days postoperatively, the majority of which were transient and self limiting.

To our knowledge, this is the first study, using a sophisticated diagnostic pacemaker device attached to bipolar epicardial atrial and ventricular leads, that has analysed the detailed onset mechanisms, durations, and temporal patterns of post-CABG AF. In particular all episodes of AF (> 15 ventricular beats) were documented by the device, which differs from previous monitoring studies. The high sensitivity and specificity of the Selection device (by comparison with external 24 hour ECGs) in reliably detecting AF episodes in community patients has recently been reported.$^{28}$ Our device's data were also corroborated by an external 24 hour ECG in a third of the AF patients.

The study results indicate that there is much intrapatient and interpatient variability in the events leading to the main AF onset, but the majority of DORs (71\%) showed preceding short runs of AF or multiple atrial extrasystoles. For all the AF episodes combined (including the short runs) conducted atrial extrasystoles accounted for most of the final triggers $(72.2 \%)$ and no trigger onsets for the rest $(27.1 \%)$, apart from one ventricular extrasystole induced onset. The onset mechanisms have shown the apparent randomness of AF initiation in this post-surgical population, with any of the preceding short runs of AF or the multiple atrial extrasystoles capable of precipitating a sustained episode. Additionally 27\% of the DORs contained just one conducted atrial extrasystole that initiated AF onset with no other antecedent arrhythmic events.

The significantly greater number of atrial extrasystoles seen on days 3 and 4 postoperatively in the AF group supports the role of atrial extrasystole in initiating AF after CABG. The external milieu (including daily normal plasma potassium concentrations of 4-5 mmol/l for all patients) was similar for each patient and so inherent individual electrophysiological differences in atrial substrate may be responsible for the increased atrial extrasystoles seen in the AF group.

It is noteworthy that no patient had any bradycardic triggers, which contrasts with community patients with PAF. Hoffmann and colleagues ${ }^{29}$ used a permanent Vitatron Selection 900 DDD pacemaker to analyse PAF onsets (in patients with drug refractory PAF) in the AFT (atrial fibrillation therapy) study. This study found that in 403 PAF onsets, $46 \%$ of the triggers were related to atrial extrasystoles, $22 \%$ to bradycardia, and $4 \%$ to a tachycardia or accelerated heart rate before the PAF onset. They also found that $71 \%$ of PAF restarts occurred within one minute compared with $46 \%$ in the present study. The relatively high sympathetic drive known to occur in the post-surgical population may account for the lack of bradycardic triggers and the predominance of atrial extrasystole and short runs of AF before the main PAF onset. Clearly, atrial pacing algorithms aimed at eliminating bradycardia would be inappropriate for this post-CABG population.

\section{AF frequency, duration, and preceding sinus rate}

The number and duration of PAF episodes in our patients were very variable. This complete variability in frequency and duration of AF episodes indicates that in this patient group, AF does not beget AF.

The average sinus rate of 99 (21) beats/min in the minute before AF onset, together with the majority of onsets being preceded by short runs or multiple atrial extrasystoles, implies that simple continuous atrial overdrive pacing algorithms, with atrial rates $<100$ beats/min, are unlikely to be effective in preventing the majority of PAF episodes in this patient group.

However, five recently published controlled studies have shown a reduction in the incidence of post-CABG AF by continuous single or dual site atrial pacing. ${ }^{16-20}$ The important postoperative use of $\beta$ blockers was similar for paced and control groups in three of these studies. ${ }^{16}{ }^{1920}$ All of these studies effectively used continuous dynamic atrial overdrive pacing algorithms.

Greenberg and colleagues $^{16}$ used a pacing algorithm dependant on the native heart rate that ensured an atrial pacing rate of $100-110$ beats/min. The paced atrial rate was adjusted whenever the native heart rate equalled or exceeded the paced rate to try to ensure continuous atrial pacing. The right atrial pacing group had a $79 \%$ reduction in the incidence of postoperative AF. In the study of Blommaert and colleagues, ${ }^{17}$ a dynamic atrial overdrive pacing algorithm was used so that right atrial pacing was continuous to an upper limit of 125 beats/min. A $63 \%$ reduction in the incidence of postoperative AF was observed. The successful study of Levy and associates ${ }^{18}$ of biatrial pacing after CABG used a specific atrial resynchronised algorithm to ensure continuous biatrial pacing. This significantly decreased the incidence of clinical post-CABG AF from $33.8 \%$ to $10.8 \%{ }^{18}$ These studies indicate that continuous dynamic atrial overdrive pacing with relatively rapid rates can significantly reduce the incidence of post-CABG AF, possibly by suppressing atrial extrasystole and dispersion of atrial refractoriness. ${ }^{19}$

Recent analysis of the phase 3 data from the AFT study further supports the role of preventive atrial pacing in $\mathrm{AF}^{30}$ In this study the preventive effect of four combined algorithms versus conventional DDD pacing at 70 beats/min was compared. A $30 \%$ reduction in AF burden (hours a day in AF) was seen with AF preventive treatment.

Our results suggest that for post-CABG AF prevention, where there are relatively high sinus rates, frequent atrial extrasystoles, and short runs of AF, a combination of the pace conditioning and atrial extrasystole suppression algorithms may be the most appropriate treatment. In pace conditioning the atrial pacing rate is dynamically adjusted to just above the underlying intrinsic rate so that the atrium is paced for at least $95 \%$ of the time. The effect is similar to the algorithms already used in the post-CABG AF pacing trials. However, atrial extrasystole suppression is aimed at reducing the incidence of atrial extrasystoles by increasing the atrial rate on detection of an atrial extrasystole. This increased rate is kept stable for a certain time period to try to prevent clusters of atrial extrasystoles.

Finally in our study it is noteworthy that $50 \%$ of the 24 patients who developed AF/AFL had episodes of only AFL ( $17 \%$ ) or both AF and AFL (33\%). This has important implications for pharmacotherapy. Digoxin appears to slow the ventricular response rate in AFL less successfully than in AF and flecainide can slow the flutter rate sufficiently to result in l:1 atrioventricular conduction and subsequent rapid ventricular rates. ${ }^{31}$

\section{Future directions}

Pharmacotherapy alone for the prevention of post-CABG AF has not proved uniformly successful. Continuous dynamic 
atrial overdrive pacing, in small trials, has also had success in reducing post-CABG AF. Combination treatment with drugs and enhanced atrial pacing algorithms now deserves further study in large randomised placebo controlled trials. Pacing targeted at the high risk elderly population ( $>65$ years) and in conjunction with $\beta$ blockade (to help reduce atrial ectopy) may be logistically and economically the most appropriate post-CABG AF preventive treatment.

\section{Study limitations}

In our study, despite the use of bipolar electrodes (Medtronic 6495), which provide much more accurate atrial sensing, atrial undersensing and far field $\mathrm{R}$ wave detection was still a problem. This was probably because the bipolar atrial electrode was not always sewn securely into the thin atrial myocardium or partially dislodged during episodes of AF. This problem has been recognised only because of the detailed diagnostic facilities available in the Selection device and other modern pacemakers. This issue needs to be addressed to avoid possible proarrhythmic atrial pacing, which is not unrecognised; in two recent studies of biatrial and right atrial pacing (using unipolar electrodes and continuous atrial pacing) AF was provoked by atrial lead undersensing. ${ }^{14} 15$

The high atrial sensitivity of $0.25 \mathrm{mV}$ in our study (necessary for reliable AF detection) may have accounted for some of the far field $\mathrm{R}$ wave detection. Pacemaker manufacturers are developing new sensing algorithms to circumvent this problem, together with shorter ring to tip spacing bipolar electrodes.

\section{Conclusions}

Our study has shown that post-CABG AF episodes are generally frequent, recurrent, and initiated by atrial extrasystoles. $\mathrm{AF}$ and AFL often occur in the same patient. Pacemaker diagnostics have shown that in the majority of patients the main episode of AF was preceded by either short runs of AF or multiple atrial extrasystoles and that the most frequent final trigger was a conducted atrial extrasystole. There was a significant increase in atrial extrasystole (in patients who developed AF) on days 3 and 4 postoperatively, which is when $86 \%$ of all AF/AFL episodes occurred. These results have major implications for the development of new preventive atrial based pacing algorithms, which in conjunction with appropriate antiarrhythmic treatment may enhance the prevention of post-CABG AF and so decrease hospital stay and costs.

\section{ACKNOWLEDGEMENTS}

We thank the cardiothoracic surgeons and the nurses on the CTICU for their help with the study; Vitatron Medical, Dieren, Netherlands for the loan of the Selection 900E pacemakers and their considerable technical help; and Dr Steve Shaw and Dr Chris Rickets, University of Plymouth, for statistical review. Vitatron Medical kindly loaned the four Selection 900E devices used in the study and provided technical help in their programming and in the analysis of the results. The study was funded by Dr A J Marshall's Research Fund.

\section{Authors' affiliations}

A D Taylor, S L Thorn, C T Lewis, A J Marshall, The South West Cardiothoracic Centre, Derriford Hospital, Plymouth, UK J G Groen, Vitatron Medical BV, 6951 KL Dieren, Netherlands

\section{REFERENCES}

1 Almassi GH, Schowalter T, Nicolosi AC, et al. Atrial fibrillation after cardiac surgery: a major morbid event? Ann Surg 1997;226:501-13.

2 Mathew JP, Parks R, Savino JS, et al. Atrial fibrillation following coronary artery bypass graft surgery. JAMA 1996;276:300-6.

3 Aranki SF, Shaw DP, Adams DH, et al. Predictors of atrial fibrillation after coronary artery surgery: current trends and impact on hospital resources. Circulation 1996;94:390-7.
4 Frost L, Molgaard H, Christiansen EH, et al. Atrial fibrillation and flutter after coronary artery bypass graft surgery: epidemiology, risk factors and preventative trials. Int J Cardiol 1992;36:253-61.

5 Fuller JA, Adams GG, Buxton B. Atrial fibrillation after coronary artery bypass grafting: is it a disorder of the elderly? J Thorac Cardiovasc Surg 1989;97:821-5.

6 Ommen SR, Odell JA, Stanton MS. Atrial arrhythmias after cardiothoracic surgery. N Engl J Med 1997;336:1429-34

7 Taylor GJ, Malik SA, Colliver JA, et al. Usefulness of atrial fibrillation as a predictor of stroke after isolated coronary artery bypass grafting. Am J Cardiol 1987;60:905-7

8 Creswell LL, Schuessler RB, Rosebloom M, et al. Hazards of postoperative atrial arrhythmias. Ann Thorac Surg 1993;56:539-49.

9 Kowey PR, Taylor JE, Rials SL, et al. Meta-analysis of the effectiveness of prophylactic drug therapy in preventing supraventricular arrhythmias early after coronary artery bypass grafting. Am J Cardiol 1992;69:963-5.

10 Gomes JA, Ip J, Santoni-Rugiu F, et al. Oral d, I sotalol reduces the incidence of postoperative atrial fibrillation in coronary artery bypass surgery patients: a randomised, double-blind, placebo-controlled study. J Am Coll Cardiol 1999;34:334-9.

11 Daoud EG, Strickberger AS, Man KC, et al. Preoperative amiodarone as prophylaxis against atrial fibrillation after heart surgery. N Engl J Med 1997;337:1785-91.

12 Guarnieri T, Nolan S, Gottlieb SO, et al. Intravenous amiodarone for the prevention of atrial fibrillation after open heart surgery: the amiodarone reduction in coronary heart (ARCH) trial. J Am Coll Cardiol 1999;34:343-7.

13 Giri S, White M, Dunn $A B$, et al. Oral amiodarone for prevention of atrial fibrillation after open heart surgery, the atrial fibrillation suppression trial (AFIST): a randomised placebo-controlled trial. Lancet 2001;357:830-6

14 Gerstenfeld EP, Hill MRS, French SN, et al. Evaluation of right atrial and biatrial temporary pacing for the prevention of atrial fibrillation after coronary artery bypass surgery. J Am Coll Cardiol 1999;33:1981-8.

15 Kurz DJ, Naegeli B, Kunz M, et al. Epicardial biatrial synchronous pacing for prevention of atrial fibrillation after cardiac surgery. Pacing Clin Electrophysiol 1999;22:721-6.

16 Greenberg MD, Katz NM, luliano S, et al. Atrial pacing for the prevention of atrial fibrillation after cardiovascular surgery. J Am Coll Cardiol 2000;35: 1416-22.

17 Blommaert D, Gonzalez M, Mucumbitsi J, et al. Effective prevention of atrial fibrillation by continuous atrial overdrive pacing after coronary artery bypass surgery. J Am Coll Cardiol 2000;35:1411-5.

18 Levy T, Fotopoulos G, Walker S, et al. Randomized controlled study investigating the effect of biatrial pacing in prevention of atrial fibrillation after coronary artery bypass grafting. Circulation 2000;102:1382-7.

19 Daoud EG, Dabir R, Archambeau M, et al. Randomized, double-blind trial of simultaneous right and left atrial epicardial pacing for prevention of post-open heart surgery atrial fibrillation. Circulation 2000;102:761-5.

20 Fan K, Lee KL, Clement SW, et al. Effects of biatrial pacing in prevention of postoperative atrial fibrillation after coronary artery bypass surgery. Circulation 2000;102:755-60

21 Leitch JW, Thomson D, Baird DK, et al. The importance of age as a predictor of atrial fibrillation and flutter after coronary artery bypass grafting. J Thorac Cardiovasc Surg 1990;100:338-42.

22 Mitchell LB. Incidence, timing and outcome of atrial tachyarrhythmias after cardiac surgery. In: Steinberg JS. ed. Atrial fibrillation after cardiac surgery. Dordrecht: Kluwer Academic, 2000:37-50

23 Cooklin M, Gold MR. Implications and treatment of atrial fibrillation after cardiothoracic surgery. Curr Opin Cardiol 1998;13:20-8.

24 Jalife J, Berenfield O, Skanes A, et al. Mechanism of atrial fibrillation: mother rotors or multiple daughter wavelets or both. J Cardiovasc Electrophysiol 1998;9:S2-12.

25 Papageorgiou P, Monahan K, Boyle NG, et al. Site-dependent intra-atrial conduction delay: relationship to initiation of atrial fibrillation. Circulation 1996;94:384-9.

26 White HD, Antman EM, Glynn MA, et al. Efficacy and safety of timolol for prevention of supraventricular tachyarrhythmias after coronary artery bypass surgery. Circulation 1984;70:479-84.

27 Crosby LH, Pifalo WB, Woll KR, et al. Risk factors for atrial fibrillation after coronary artery bypass grafting. Am J Cardiol 1990;66:1520-2.

28 Carlioz R, Perrier E, Thomas O, et al. Accuracy of atrial tachyarrhythmia monitoring in the Selection device: correlation with an external Holter recording. In: Bloch Thamsen PE, ed. Eurospace 2001. Bologna, Italy: Monduzzi Editore, 2001:419-23.

29 Hoffmann E, Janko S, Steinbeck G, et al. Analysis of onset mechanisms of atrial fibrillation in pacemaker patients. Heart 2000:83(suppl 1):19.

30 Camm AJ, on behalf of the AFtherapy study group. The atrial fibrillation therapy study. European Society of Cardiology XXIII Congress, Hot Line II (http://www.escardio.org/congress/Stock01/hotline/slides/ aftherapy.pdf). Stockholm, 1-5 September 2001.

31 Braunwald E, ed. Heart disease: a textbook of cardiovascular medicine, 6th edn. Phildelphia: WB Saunders, 2001:832. 